\title{
PRIMARY SJÖGREN'S ENCEPHALITIS: A CASE REPORT
}

\author{
Caroline Vargas da Cruz', Letícia Serrano Sartori', Dawton Yokito Torigoe', Juliana D’Agostino Gennari', Francisco Tomaz \\ Meneses de Oliveira ${ }^{1}$
}

1.Santa Casa de Misericórdia de São Paulo, São Paulo (SP), Brazil.

*Corresponding author: carolvargascruz@hotmail.com

\section{BACKGROUND}

Sjögren's syndrome (SS) is a systemic disease of little known etiophysiopathogenesis, which predominantly affects women. It is characterized by lymphocytic infiltration in the exocrine glands and less frequently, by involvement of organs and systems. It can be primary (SSp) if it occurs alone or secondary (SSs) when in association with other autoimmune diseases such as rheumatoid arthritis, systemic lupus erythematosus and systemic sclerosis. Glandular involvement, mainly salivary and lacrimal, is responsible for xerophthalmia and xerostomia (sicca symptoms), the most common manifestations and present in up to $98 \%$ of cases. Systemic manifestations occur in the form of arthralgia or polyarthritis, interstitial lung disease, skin lesions, tubulointerstitial nephritis or glomerulonephritis. Neurological involvement is also relevant; it is considered a rare and uncommon extraglandular manifestation, which can occur even in the absence of sicca symptoms.

\section{CASE REPORT}

Female patient, 41 years old, white, hypertensive, admitted to a hospital for paraparesis and hypoaesthesia of crural predominance associated with double incontinence and low acuity of vision in the left eye started 15 days ago. The skull magnetic resonance imaging (MRI) showed T2/FLAIR hypersignal in the posterior aspect of the bulb and proximal spinal cord segment including posterior area and fundscopy with loss of differentiation of the optic disc margins on the left, favoring the diagnosis of longitudinally extensive demyelinating disease, of the spectrum of the neuromyelitis optica. We opted for the investigation for possible rheumatology, with discrete sicca symptoms, arthralgia and fatigue being identified. Antinuclear factor (ANF) 1/80 fine dotted nuclear pattern, antiSSA and anti-SSB in high titers and minor salivary gland biopsy with chronic sialoadenitis, liposubstitution and 1 focus / scores. The diagnosis of SSp with neurological manifestations was made and methylprednisolone $1 \mathrm{~g}$ was started. There was no response after the third day of treatment, and due to the severity of the condition, plasmapheresis was performed followed by infusions of rituximab with good response to treatment.

\section{CONCLUSION}

The neurological manifestations of SSp are broad and affect the peripheral nervous system more frequently. The involvement of the central nervous system still remains controversial, possibly due to uncertain pathophysiological knowledge, due to the different classification criteria and the variation in prevalence between studies, which makes this type of involvement little related to SSp. Primary Sjögren's syndrome should be considered in the presence of an unexplained motor neuron syndrome or CNS involvement, even in the absence of sicca symptoms. Thus, diagnosis and treatment can be made early, impacting the prognosis of the disease. 